\title{
PROYECTO DE SISTEMAS DE CUIDADO ESENCIAL EN TRAUMA
}

\author{
Essential Trauma Care Project
}

Andrés M. Rubiano Escobar. Investigador Asociado, Departamento de Cirugía, Centro Médico de la Universidad de Pittsburgh (PA) - USA

Correspondencia: e-mail: rubianoam@upmc.edu

\section{RESUMEN}

Los sistemas de atención de trauma con centros especializados, fueron establecidos en servicios de salud de países de alto ingreso hace más de 30 años. La efectividad de estos centros, ha sido evaluada con resultados que muestran un beneficio en los índices de sobrevida y minimización de las secuelas post trauma, de los pacientes atendidos en ellos. La importación directa de estos modelos, muchas veces no es posible en países de mediano y bajo ingreso, ya que su implementación requeriría una disponibilidad de recursos con la que no se cuenta en estos sitios. Un enfoque basado en la evidencia brindada por estos estudios de resultados, favorables pero acomodado a la realidad de la salud pública de cada país, es necesario con el ánimo de brindar mejores oportunidades a las víctimas de lesiones traumáticas a nivel global. Este artículo presenta una revisión sobre el proyecto de las Guías esenciales de Trauma de la Organización Mundial de la Salud y las ventajas de este enfoque para su aplicación en países como Colombia.

\section{PALABRAS CLAVE}

Sistemas de Trauma, Trauma, Centro de Trauma, Guías de Trauma

\section{ABSTRACT}

Trauma treatment systems in specialized centers were established in health services in high-income countries more than 30 years ago. The effectiveness of these centers has been evaluated. Their results show an increase in patients' survival rates and a minimization in post-trauma after effects Most of the times, direct import of these models is not possible in low and middle income countries, because their implementation would require availability of economic resources that is not affordable in these countries. An approach based on the evidence showed by these studies with positive results, but adjusted according to the reality of public health in each one of these countries, is necessary. It is aimed at giving better opportunities to the traumatically-injured people worldwide. This article presents a review of the Essential Trauma Care Project of the World Health Organization, and the approach advantages if used in countries like Colombia.

\section{KEY WORDS}

Trauma Systems, Trauma, Trauma Center, Trauma Guidelines 


\section{INTRODUCCIÓN}

La categorización de los países por nivel económico, se encuentra basada en el valor del ingreso bruto nacional per cápita (tabla $\mathbf{1}$ ).

Tabla 1. Clasificación de los países de acuerdo a su ingreso anual per cápita.
CLASIFICACIÓN

BAJO INGRESO

BAJO-MEDIANO INGRESO

ALTO-MEDIANO INGRESO

ALTO INGRESO
INGRESO ANUAL PER CAPITA (GNI)

$<735^{*}$

$736-2.395$

$2.396-9.075$

$>9.076$
*Valores en dólares americanos, datos del Banco Mundial.

Muchos de los países latinoamericanos, no sobrepasan los US\$736 dólares, clasificándose dentro de los de bajo y bajo mediano ingreso, en donde la disponibilidad del presupuesto asignado a salud es mínimo y la disponibilidad de recursos especialmente en áreas remotas o rurales, no permite brindar un cuidado avanzado de patologías complejas. Colombia actualmente se considera un país de Bajo-Mediano ingreso, con un promedio de US\$2.100 dólares anuales per cápita (US\$6 dólares diarios), pero el $17 \%$ de la población está con ingresos promedio de US $\$ 2$ dólares y el $7 \%$ presenta ingreso diarios menores a $\$ 1$ USD. Este $24 \%$, es solo una parte de la población total, que recibe su atención en instituciones públicas de salud1. El trauma es una enfermedad predominante en la población joven, su incidencia es mas alta y a la vez sus consecuencias son más devastadoras, en países de bajo y mediano ingreso, generalmente asociado a diversas condiciones como menor disponibilidad de recursos para su cuidado y además políticas leves de prevención1.

Basados en esta realidad, la organización de los recursos actualmente disponibles para el cuidado del trauma, es muy importante si se quiere enfrentar con éxito el manejo de patologías criticas como esta, tan importantes en nuestro medio. Para tener una idea sobre la intensidad del problema en países de mediano-bajo ingreso, en Colombia por ejemplo para el 2007, se registraron 282.457 lesiones traumáticas (registradas por medicina legal) y el $47 \%$ de estas estuvieron relacionadas con violencia interpersonal. Para este mismo año se registraron 27.920 muertes violentas y el 59\% de estas fueron homicidios.2 Para conocer cifras locales, por ejemplo, en el año 2008, el Hospital Universitario de Neiva, realizo aproximadamente 4.445 atenciones de urgencias a pacientes con algún tipo de lesión por trauma, muchas de estas requiriendo hospitalización y manejo médico- quirúrgico. De esta forma es claro, que el trauma, ha sido una patología de alta incidencia en nuestro medio y ha sido la primera causa de muerte e invalidez en Colombia hasta hace una par de años, en los cuales, la enfermedad cardiovascular ha superado las tasas de morbi-mortalidad del trauma.

De acuerdo a los reportes del 2006 del Banco Mundial, Colombia invierte en salud 7,6\% del Producto Interno Bruto y existen alrededor de 1,3 médicos por cada 1.000 habitantes, con una disponibilidad de camas hospitalarias de 1,1 por cada 1.000 habitantes3. La reflexión que debemos hacer dentro de este contexto, es cuántos de estos médicos y cuántas de estas camas disponibles, están ubicados en centros que cuentan con la infraestructura y el entrenamiento apropiado para el cuidado avanzado del trauma. Dentro de los pocos estudios realizados en países de mediano y bajo ingreso, sobre la calidad de atención del trauma, se reportan cifras tan exorbitantes; como mortalidades entre 6 y 12 veces mayores en pacientes politraumatizados de acuerdo a las características y los recursos del centro en donde fueron atendidos4. Esto quiere decir, que si en nuestro medio, un paciente traumatizado grave, es trasladado a un centro asistencial que no cuenta con los recursos apropiados, tiene entre 6 y 12 veces más de posibilidades de fallecer, por fallas en el proceso de atención.

\section{PROYECTO DE GUÍAS DE CUIDADO ESENCIAL EN TRAUMA}

Debido a esto, la Organización Mundial de la Salud, reunió en el año 2000, un comité de expertos en cuidados del trauma, con representación de todas las 
áreas del mundo, incluyendo países de alto, mediano y bajo ingreso. La esencia de este proyecto, fue el establecer recomendaciones mínimas, que se deben cumplir en una institución de salud (independiente del nivel de complejidad), para brindar un cuidado apropiado del paciente víctima del traumal. A estas recomendaciones, determinadas por opiniones de expertos y basadas en evidencia científica, se les denomino: cuidado esencial en trauma. Estas guías de cuidado esencial, se basan adicionalmente en políticas de salud reales, que puedan ser aplicadas en la mayoría de los países, independientemente de las características del nivel de ingreso y de la complejidad del sistema. La mejor alternativa para cumplir con estos requisitos, es la búsqueda de intervenciones sencillas, con el menor tipo de tecnología disponible, pero que realicen una gran diferencia, a través de la mejoría en la organización e implementación de los manejos, especialmente relacionado con las tasas de sobrevida y la disminución de las secuelas1, 5-7.

\section{METODOLOGÍA DE LAS GUÍAS}

Las 3 principales consideraciones para definir la intervención son1:

\section{RECURSO HUMANO}

El perfil del entrenamiento enfocado a la atención del trauma, debe incluir el comportamiento dentro de ambientes urbanos y rurales. Los médicos generales que atiendan trauma complejo, en niveles básicos de atención, deben tener un entrenamiento mínimo de destrezas para el manejo del trauma. La rotación de los médicos en formación de servicios de atención en trauma, al igual que el del personal de enfermería, es fundamental.

\section{RECURSOS FÍSICOS}

La mayoría de hospitales disponen de escasos recursos para la atención del trauma. Los hospitales de mayor afluencia de pacientes con trauma, deben tener elementos mínimos de insumos esenciales, para la atención de este tipo de pacientes; como son los recursos para manejo de vía aérea, la disponibilidad de tubos de tórax, etc. Se deben asegurar los recursos presupuestales para el mantenimiento permanente de equipos básicos como los escanógrafos, en los centros de referencia de neurotrauma.

\section{RECURSOS DE PROCESOS DE ORGANIZACIÓN Y ADMINISTRACIÓN}

Los tiempos de atención, la disponibilidad de recursos, de personal y la implementación de programas de aseguramiento de calidad en la prestación de los servicios, son fundamentales para lograr el éxito final del proceso. Los gobiernos nacionales, regionales y locales, deben buscar formas de implementación de políticas reales, adaptadas a sus propios recursos para una adecuada funcionalidad las 24 horas, los 365 días del año.

Existen tres principios básicos denominados, los "requerimientos del paciente de trauma", que es un concepto extrapolable a los derechos mínimos, que tiene un paciente que es atendido por trauma 1,8 Estos principios son:

1. Las lesiones que amenazan la vida, deben ser apropiadamente tratadas, de manera rápida y de acuerdo a las prioridades recomendadas, para maximizar la posibilidad de sobrevida.

2. Las lesiones potencialmente inhabilitadoras, deben ser tratadas apropiadamente, para minimizar el compromiso funcional y maximizar el retorno a la independencia funcional y a la participación en la vida comunitaria.

3. El dolor y el sufrimiento psicológico, deben ser minimizados.

Los procesos por los cuales se lleve al cumplimiento de estos "derechos", pueden variar de país en país, pero lo que no se puede justificar, es que estén sujetos al nivel de ingreso de la institución que presta los servicios. Para fines de aplicación de las recomendaciones de cuidado esencial, los sitios de atención médica se clasificaron en diferentes tipos de complejidad, cuya denominación puede variar de país en país pero, que generalmente se agrupan de la siguiente forma: Hospital Básico o Rural, Hospital Regional General, Hospital de Especialidades Básicas y el Hospital Terciario o de Alta Especialidad (Sub Especializado). Para cada uno de ellos se definieron algunos recursos que de acuerdo al nivel de atención, se catalogan como Esenciales, Deseables, Posiblemente Requeridos o Irrelevantes. De esta forma, se elaboraron tablas de recomendaciones, que incluyen perfiles de entrenamiento, mejoría de rendimiento, organización de sistemas de trauma y planes de organización hospitalaria, que deben influir de manera positiva en los modelos de atención de trauma de manera local, regional y nacional. 
La matriz de elaboración del proyecto, se basa en los 4 grandes grupos de centros asistenciales presentes en todos los países (Tabla 2):

Tabla 2. Caracterización de los centros de asistencia en trauma aplicables a nivel mundial.

\begin{tabular}{|c|c|c|c|}
\hline \multicolumn{4}{|c|}{ CENTROS DE CUIDADOS } \\
\hline BÁSICOS & GENERALES & ESPECIALIZADOS & TERCIARIOS \\
\hline $\begin{array}{l}\text { PUESTOS DE SALUD } \\
* \text { (NIVEL I) }\end{array}$ & $\begin{array}{l}\text { HOSPITALES CON MÉDICOS } \\
\text { GENERALES }\end{array}$ & $\begin{array}{l}\text { HOSPITALES CON } \\
\text { CIRUJANOS GENERALES }\end{array}$ & $\begin{array}{l}\text { FACILIDADES DE CUIDADOS } \\
\text { TERCIARIOS CON LIMITADAS } \\
\text { ESPECIALIDADES }\end{array}$ \\
\hline $\begin{array}{l}\text { CLÍNICAS CON } \\
\text { ENFERMERAS Y } \\
\text { PERSONAL PARAMÉDICO }\end{array}$ & $\begin{array}{l}\text { HOSPITALES CON MÉDICOS } \\
\text { GENERALES Y CAPACIDAD } \\
\text { QUIRÚRGICA } \\
\text { *(NIVEL II) }\end{array}$ & $\begin{array}{l}\text { HOSPITALES CON } \\
\text { CIRUJANOS GENERALES } \\
\text { Y ORTOPEDISTAS }\end{array}$ & $\begin{array}{l}\text { FACILIDADES DE CUIDADOS } \\
\text { TERCIARIOS CON TODAS } \\
\text { LAS ESPECIALIDADES } \\
\text { *(NIVEL IV) }\end{array}$ \\
\hline CLÍNICAS CON MÉDICOS & & $\begin{array}{l}\text { HOSPITALES CON } \\
\text { CIRUJANOS GENERALES, } \\
\text { ORTOPEDISTAS Y OTROS } \\
\text { ESPECIALISTAS } \\
\text { *(NIVEL III) }\end{array}$ & \\
\hline
\end{tabular}

${ }^{*}$ Equivalencia de centros de atención aplicable al modelo de salud Colombiano, datos de ETC Project (WHO) 2004.

Esta misma matriz, determina 4 tipos de recursos que deben o no, estar disponibles en cada uno de los diferentes centros de acuerdo a su nivel de complejidad, independiente del nivel de recursos de cada región (Tabla 3):

Tabla 3. Caracterización de los tipos de recursos disponibles para el manejo del trauma, aplicables a nivel mundial.

\begin{tabular}{c|c}
\multicolumn{2}{c}{ RECURSOS* } \\
\hline TIPOS & CARACTERÍSTICAS \\
\hline
\end{tabular}
ESENCIALES común, de todos los sistemas de trauma, en todas las regiones del mundo, incluyendo países con inversiones de tan solo us $\$ 3$ a 4 dólares per cápita para salud por año.

Son recursos que representan una capacidad de mejorar, la probabilidad de éxito en la atención del traumatizado, adicionando un costo por encima de lo presupuestado. No son elementos costo efectivos en todos los niveles y por

DESEABLES eso mismo no se consideran esenciales. Cada país debe evaluar la posibilidad de incluirlos en sus propios planes nacionales de presupuesto de salud. De acuerdo al volumen de trauma de cada país, algunos de estos elementos pueden volverse esenciales.

En algunas áreas de extremada pobreza, algunos elementos para el tratamiento del trauma deben ser distribuidos en

POSIBLEMENTE REQUERIDOS

IRRELEVANTES centros de menor complejidad, por su escasa disponibilidad. Esto lleva, a que personal sin el entrenamiento apropiado deba hacer uso de estos recursos que en centros de mayor disponibilidad son realizados por personal más preparado. Estos son recursos que pueden de alguna manera, mejorar la disponibilidad de tratamientos inmediatos en áreas remotas y, se encuentra enfocado especialmente a países de bajos ingresos.

Son recursos que no necesariamente deben estar, en el centro de atención de acuerdo a su nivel de complejidad, aunque se tenga una alta disponibilidad de recursos en cada nivel. 
Ejemplo: recomendaciones específicas neurotrauma

Mas del $65 \%$ de la mortalidad en neurotrauma, se asocia a lesión cerebral secundaria, por fallas en el proceso de oxigenación cerebral y en el mantenimiento de presiones arteriales adecuadas y por tanto una recomendación esencial, es reforzar el proceso de manejo del $\mathrm{ABC}$ inicial con destrezas y equipos adecuados de acuerdo al nivel, para manejo en de vía aérea (A), adecuada respiración y ventilación (B) y un optimo estado hemodinámico (C). Son desafortunadamente frecuentes los casos de lesiones traumáticas cerebrales leves o moderadas, agravadas por compromiso ventilatorio o hemodinámico.

(Figura 1) Paciente pediátrico con TCE moderado, que se deteriora durante la fase de atención inicial, en una institución de Nivel I rural por imposibilidad de manejar la vía aérea. El TAC cerebral de ingreso

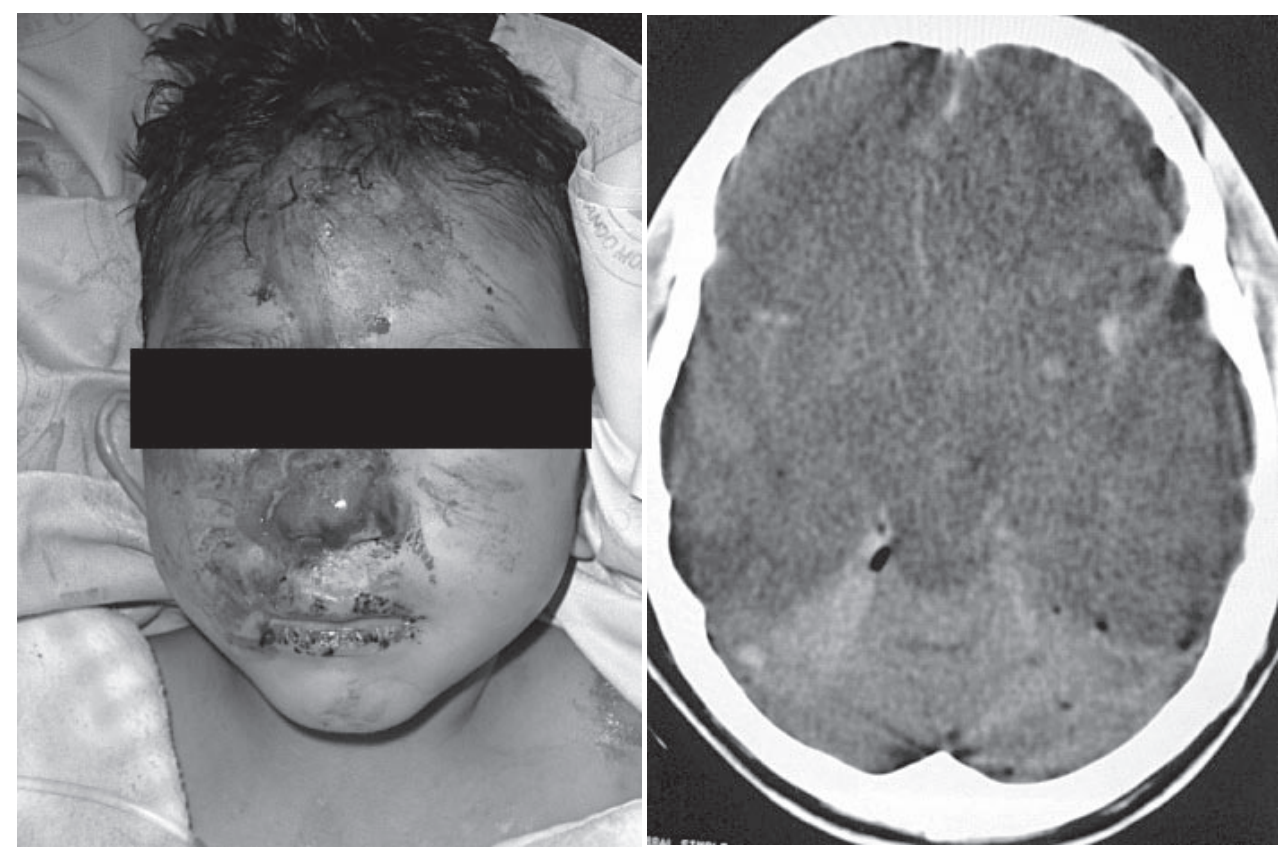

Figura 1. Tomografía axial computarizada de paciente pediátrico tomada durante la fase de atención inicial.

al centro de remisión (luego de 90 minutos), evidencia un compromiso isquémico global asociado a hipoxia cerebral. (Imagen: Autor)

Un ejemplo de una de las tablas de recomendaciones específicas, se presenta a continuación (Tabla 4)

Tabla 4. Caracterización de los recursos para manejo del Trauma Craneoencefálico de acuerdo al nivel de complejidad de la atención.

\begin{tabular}{l|c|c|c|c|}
\multirow{2}{*}{\multicolumn{1}{c|}{ TIPO DE RECURSO }} & \multicolumn{3}{|c|}{ TIPOS DE CENTROS DE CUIDADOS } \\
\cline { 2 - 5 } & BASICO & GENERAL & ESPECIALIZADO & TERCIARIO \\
\hline $\begin{array}{l}\text { RECONOCIMIENTO DE ALTERACIÓN DE CONCIENCIA, } \\
\text { SIGNOS DE LATERALIZACIÓN Y ALTERACIÓN PUPILAR. }\end{array}$ & E & E & E & E \\
\hline $\begin{array}{l}\text { SEGUIMIENTO COMPLETO DE LAS GUÍAS PARA MANEJO } \\
\text { DEL TRAUMA CRANEOENCEFÁLICO SEVERO DE LA BTF/ } \\
\text { AANS. }\end{array}$ & $\mathrm{I}$ & $\mathrm{I}$ & $\mathrm{D}$ & $\mathrm{D}$ \\
\hline $\begin{array}{l}\text { MANTENIMIENTO DE OXIGENACIÓN Y NORMO TENSIÓN } \\
\text { PARA PREVENIR LESIÓN CEREBRAL SECUNDARIA. }\end{array}$ & $\mathrm{D}$ & $\mathrm{E}$ & $\mathrm{E}$ & E \\
\hline
\end{tabular}




\begin{tabular}{|c|c|c|c|c|}
\hline \multirow{2}{*}{ TIPO DE RECURSO } & \multicolumn{4}{|c|}{ TIPOS DE CENTROS DE CUIDADOS } \\
\hline & BASICO & GENERAL & ESPECIALIZADO & TERCIARIO \\
\hline $\begin{array}{l}\text { EVITAR SOBRE-HIDRATACIÓN EN PACIENTES CON PIC } \\
\text { ELEVADA (CON PAS NORMAL). }\end{array}$ & $\mathrm{D}$ & $E$ & $E$ & $E$ \\
\hline MONITORIA Y TRATAMIENTO DE PIC ELEVADA. & I & 1 & D & D \\
\hline TAC CEREBRAL. & I & D & D & D \\
\hline $\begin{array}{l}\text { BURR HOLES (EQUIPO Y ENTRENAMIENTO PARA } \\
\text { ORIFICIOS DE TREPANACIÓN). }\end{array}$ & 1 & PR & $\mathrm{D}$ & $E$ \\
\hline $\begin{array}{l}\text { PROCEDIMIENTOS NEUROQUIRURGICOS AVANZADOS } \\
\text { (DESCOMPRESIÓN). }\end{array}$ & I & I & PR & D \\
\hline $\begin{array}{l}\text { TRATAMIENTO QUIRÚRGICO DE FRACTURAS DE CRÁNEO } \\
\text { DEPRIMIDAS ABIERTAS. }\end{array}$ & I & PR & D & $\mathrm{E}$ \\
\hline $\begin{array}{l}\text { TRATAMIENTO QUIRÚRGICO DE FRACTURAS DE CRÁNEO } \\
\text { DEPRIMIDAS CERRADAS. }\end{array}$ & 1 & 1 & PR & D \\
\hline $\begin{array}{l}\text { MANTENIMIENTO DE REQUERIMIENTOS PROTEICOS Y } \\
\text { CALÓRICOS. }\end{array}$ & 1 & $\mathrm{E}$ & $\mathrm{E}$ & $\mathrm{E}$ \\
\hline
\end{tabular}

*(BTF: Brain Trauma Foundation, AANS: American Association of Neurological Surgeons, PIC: Presión Intracraneana, PAS: Presión Arterial Sistólica, TAC: Tomografía Axial Computarizada). $E=$ Recurso esencial; $D=$ Recurso deseable; $P R=$ Recurso posiblemente requerido, I= Recurso irrelevante, datos ETC Project (WHO) 2004.

Es la tabla para las guías de manejo de lesiones craneales a nivel de cada uno de los diferentes tipos de centros de atención, de acuerdo a la matriz de destrezas presentada en la tabla 3.

Es importante tener en cuenta, para la interpretación de estas recomendaciones, que estas son genéricas, y por tanto tienen implicaciones diferentes en cada país. Por ejemplo, en algunos países (especialmente de África y Asia) no existe disponibilidad de un neurocirujano en centros de cuidado terciario, especialmente en áreas remotas y por tanto algunos procesos de entrenamiento allí, buscan involucrar personal de urgencias y cirugía general en la realización de procedimientos de trepanación de urgencia; Si esto no se realiza, simplemente en esas áreas los pacientes fallecerían por causas prevenibles o tratables, esperando un neurocirujano que nunca va a llegar.

Respecto a las lesiones espinales, las recomendaciones se basan en una adecuada evaluación de la función motora y sensitiva, adecuadas técnicas de permeabilización de la vía aérea y ventilación, hasta el evitar las complicaciones más frecuentes en la fase de rehabilitación, como ulceras de presión, infecciones urinarias, trombosis venosa, etc., que se pueden evitar con maniobras tan sencillas como los adecuados cambios de posición al menos cada 2 horas (Tabla 5).

Tabla 5. Caracterización de los recursos para manejo del Trauma Raquimedular de acuerdo al nivel de complejidad de la atención. (AANS: American Association of Neurological Surgeons).

\begin{tabular}{|c|c|c|c|c|}
\hline \multirow{2}{*}{ TIPO DE RECURSO } & \multicolumn{4}{|c|}{ TIPOS DE CENTROS DE CUIDADOS } \\
\hline & BASICO & GENERAL & ESPECIALIZADO & TERCIARIO \\
\hline $\begin{array}{l}\text { EVALUACIÓN Y RECONOCIMIENTO DE PRESENCIA O RIESGO DE } \\
\text { LESIÓN ESPINAL. }\end{array}$ & $E^{*}$ & $\mathrm{E}$ & $E$ & $\mathrm{E}$ \\
\hline $\begin{array}{l}\text { INMOVILIZACIÓN CON COLLAR CERVICAL } \\
\text { Y TABLA ESPINAL. }\end{array}$ & D & $\mathrm{E}$ & $E$ & $E$ \\
\hline MONITORIA DE LA FUNCIÓN NEUROLÓGICA. & $\mathrm{E}$ & $\mathrm{E}$ & $\mathrm{E}$ & $\mathrm{E}$ \\
\hline $\begin{array}{l}\text { EVALUACIÓN CON UN SISTEMA DE CLASIFICACIÓN } \\
\text { INTERNACIONAL. }\end{array}$ & I & I & D & $E$ \\
\hline
\end{tabular}




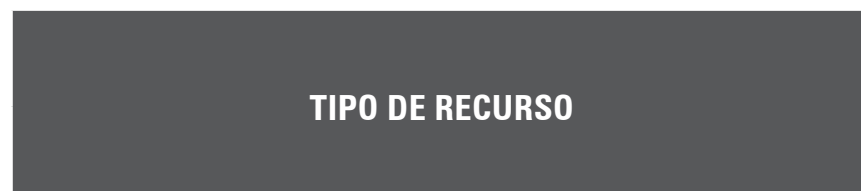

MANTENIMIENTO DE OXIGENACIÓN Y NORMO TENSIÓN PARA PREVENIR LESIÓN ESPINAL SECUNDARIA.

ABORDAJE HOLISTTICO PARA PREVENIR COMPLICACIONES, ESPECIALMENTE ULCERAS DE PRESIÓN Y RETENCIÓN E INFECCIÓN URINARIA.

TOMOGRAFÍA AXIAL COMPUTARIZADA

RESONANCIA MAGNÉTICA

SEGUIMIENTO COMPLETO DE LAS GUÍAS PARA MANEJO DE LAS LESIONES ESPINALES AGUDAS DE LA AANS.

MANEJO NO QUIRÚRGICO DEL TRM (EN CASO DE INDICACIÓN)

MANEJO QUIRÚRGICO DE LA LESIÓN ESPINAL.

MANEJO QUIRÚRGICO DE LA LESIÓN ESPINAL EN CASO DE DETERIORO POR COMPRESIÓN ESPINAL. (DESCOMPRESIÓN).

\begin{tabular}{|c|c|c|c|}
\hline \multicolumn{5}{|c|}{ TIPOS DE CENTROS DE CUIDADOS } \\
\hline BASICO & GENERAL & ESPECIALIZADO & TERCIARIO \\
\hline D & E & E & E \\
\hline D & E & E & E \\
\hline I & D & D & D \\
\hline I & I & D & D \\
\hline I & I & D & D \\
\hline I & PR & E & E \\
\hline I & I & PR & E \\
\hline I & I & PR & E \\
\hline
\end{tabular}

${ }^{*} E=$ Recurso esencial; $D=$ Recurso deseable; $P R=$ Recurso posiblemente requerido, $I=$ Recurso irrelevante, fuente ETC Project (WHO) 2004.
Intervenciones tan simples como el organizar listas de chequeo a nivel de quirófanos, servicios de urgencias y de cuidados intensivos antes de realizar procedimientos, han sido evaluadas recientemente y su aplicación incide sobre la mejoría en la sobrevida de los pacientes críticos9. Igualmente, las evaluaciones del beneficio de los centros de atención especializada en trauma, son claras y este beneficio no puede ser negado por falta de organización de las zonas y de los centros con menor disponibilidad de recursos10-12 Este proyecto debe acompañarse de un proceso de diseminación, por parte de las sociedades científicas regionales y locales y a la vez ser usadas como elementos mínimos para el desarrollo de procesos de habilitación de instituciones prestadoras de servicios de salud, que se encuentran actualmente manejando pacientes victimas de trauma. La segunda fase, debe conducir a la realización de estudios epidemiológicos y clínicos, que permitan a través de apropiadas bases de datos, evaluar si existen mejorías en los resultados, luego de la implementación de las guías de manejo10-12

\section{CONCLUSIÓNES}

El proyecto de Guías de Cuidado esencial de la Organización Mundial de la Salud, brinda unas herramientas sencillas y prácticas que involucran guías tanto prehospitalarias como intra-hospitalarias de manejo del paciente politraumatizado, para ser utilizadas en centros asistenciales de países de diferentes niveles de economía. Estas herramientas, permiten organizar apropiadamente destrezas que son fundamentales para mejorar la sobrevida de los pacientes victimas de trauma. Colombia, y especialmente regiones como Neiva, de acuerdo a su desarrollo y disponibilidad de servicios de salud, son áreas ideales para la implementación y posterior evaluación de este tipo de proyectos, que pueden generar un beneficio importante en la población usuaria, víctima de las lesiones intencionales y no intencionales.

\section{REFERENCIAS BIBLIOGRÁFICAS}

Mock C, Lormand J, Goosen J, Joshipura M, Peden M; WHO. World Health Organization, Guidelines for Essential Trauma Care. International association for the surgery of trauma and intensive care, international society of surgery. Geneva. 2004.

INMLCF: FORENSIS. Informe estadístico del instituto nacional de medicina legal y ciencias forenses. 2007.

Hsiao W, Shaw R. Social health insurance for developing nations. WBI Development Studies, The World Bank, Washington DC. 2006.

Mock CN, et al; Trauma mortality patterns in three nations at different economic levels: implications for 
global trauma system development. The Journal of Trauma. 1998; 44:804-814

Mock C, Joshipura M, Goosen J, Maier R; Overview of the essential trauma care project. World Journal Surgery. 2006; 30: 919-929.

Goosen J, Morris P, Kobusingye Or, Mock C; Advancing essential trauma care through to partner organizations: IATSIC, ISS-SIC and WHO. World Journal Surger. 2006; 30: 940-945.

Nakahara S, Ichikawa M, Kimura A, Yoshida K; The potential for essential trauma care to empower communities and tackle inequities. World Journal Surgery. 2008; 32(6):1203

American college of surgeons committee on trauma: Resources for the optimal care of the injured patient. American College of Surgeons. Chicago. 1999.

Haynes AB, Weiser TG, Berry WB, Lipsitz SR, Breizat AS, Dellinger EP et al; A surgical safety checklist to reduce morbidity and mortality in a global population. The New England Journal of Medicine. (NEJM). 2009; 360: (5): 491-500.

Nathens To, Jurkovich G, Maier R, Grossman D, MacKenzie and, Moore M, Rivara F; Relationship and between trauma to center volume outcomes. The Journal of the American Medical Association. (JAMA). 2001; 285: 1164-1171.

Mackenzie EJ, Hoyt DB, Sacred JC, et al; National inventory of hospital trauma centers. The Journal of the American Medical Association. (JAMA). 2003; 289: 1515-1522.

Nathens AB, Maier RV; The relationship between trauma to center volume and outcome. Adv Surgery. 2001; 35: 61-75. 